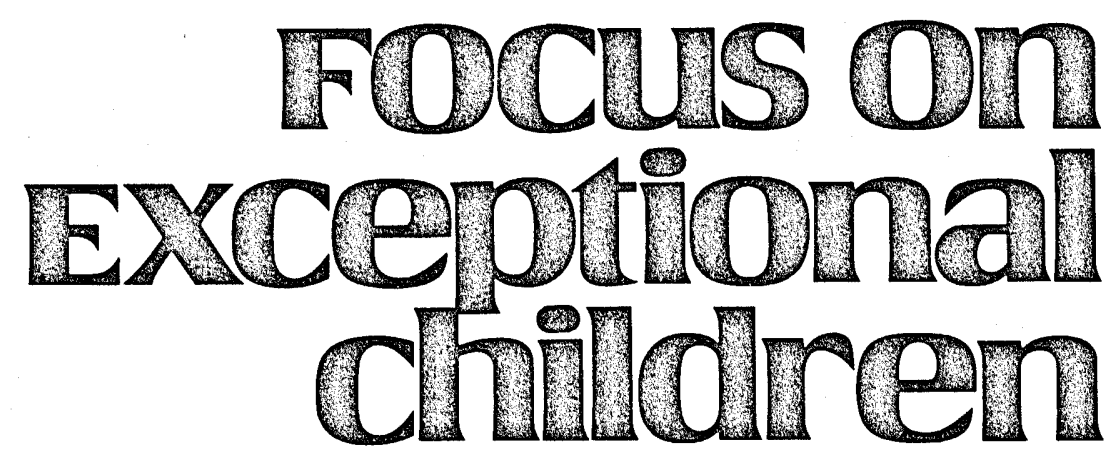

\title{
Measuring Adaptive Behavior: Issues and Instruments
}

\author{
Ronald L. Taylor
}

In the broadest sense, adaptive behavior refers to an individual's ability to cope or deal effectively with personal and social demands. Although many formal definitions of adaptive behavior have been set forth, the definition proposed by the American Association on Mental Deficiency (AAMD) is perhaps the most widely accepted: ". . . the effectiveness with which the individual meets the standards of personal independence and social responsibility" (Grossman, 1983, p.1). In a review of the various definitions and interpretations of adaptive behavior, Witt and Martens (1984) noted that most considered adaptive behavior as being age- and culture-specific and included areas such as independent functioning, social responsibility, and cognitive development.

Regardless of the formal definition used, one caveat regarding adaptive behavior is necessary: It is a difficult construct to operationalize and, subsequently, to measure. For example, some conceptions of adaptive behavior include academic and more cognitively oriented skills, whereas others intentionally omit these types of behavior. Also, as previously noted, adaptive behavior is age-specific and, to a certain extent, culture-specific. Adaptive behavior for a 3-year-old child might include skills such as eating and toileting; for an adult it might include behaviors related to social and economic responsibility.

The AAMD provides general categories of adaptive behavior skills for preschoolers, children and early adolescents, and late adolescents and adults (Grossman, 1983). Preschool skills include sensorimotor, communication, and self-help skills. Childhood and early adolescent skills are aimed primarily at practical application of academic skills and appropriate interpersonal functioning. Late adolescent and adult skills focus largely on community, vocational, and economic responsibilities.

The culture-specific nature of adaptive behavior relates not as much to the general skills expected of individuals but, rather, to the specific nature of the expected skills. For example, Reschly (1984) noted that "crossing traffic properly with a traffic light" is an important and reasonable behavior to expect from school-age children and could fall into the general category of using judgment to cope effectively with the environment. If, however, a child is from a rural area that has no traffic lights, the nature of that skill is culture-specific.

Difficulties in identifying universal, specific skills make the measurement or assessment of these skills problematic at best. Before the instruments used to measure adaptive behavior can be analyzed, however, the history and purported uses of adaptive behavior assessment should be discussed, to shed additional light on this somewhat confusing area. 


\section{THE HISTORY OF}

\section{ADAPTIVE BEHAVIOR ASSESSMENT}

Adaptive behavior is not a new concept in special education, although it only recently has gained its present level of attention-partly as a result of litigation and legislation related to the identification of mentally retarded students. In the 1970s several noteworthy court cases (e.g., Guadalupe v. Tempe, Arizona School District, 1972) brought attention to the inadequate and inappropriate assessment methods used to identify mildly retarded students. In part, sole use of IQ in determining eligibility for special education was criticized because of the alleged bias of IQ scores against minority children. The result was a disproportionate number of ethnic minority children in classes for mildly retarded students.

In the early 1970 s Mercer, a leading proponent of the adaptive behavior movement, characterized this phenomenon by referring to many of these students as "six-hour retarded children" (Mercer, 1973). This characterization implied that although they had difficulty during the 6 hours spent in the school environment, these children were able to meet demands required in other environments. Phrased another way, these students were not deficient in adaptive behavior skills.

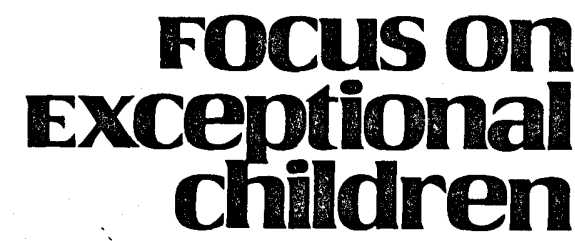

FOCUS ON EXCEPTIONAL CHILDREN (ISSN0015-511X) (USPS 203-360) is published monthly except June, July, and August as a service to teachers, special educators, curriculum specialists, administrators, and those concerned with the special education of exceptional children. This journal is abstracted and indexed in Exceptional Child Education Resources, and is also available in microform from Xerox University Microfilm. Ann Arbor, Michigan. Subscription rates, $\$ 18.00$ per year. Copyright $\odot 1985$, Love Publishing Company. All rights reserved. Reproduction in whole or part without written permission is prohibited. Printed in the United States of America. Second class postage is paid at Denver, Colorado.

POSTMASTER: Send address changes to:

Love Publishing Company

Executive and Editorial Office

1777 South Bellaire Street

Denver, Colorado 80222

Telephone (303) 757-2579

EDITORIAL BOARD
Edward L. Meyen

University of Kansas

Richard $\mathbf{J}$, Whelan

University of Kansas Medical Center
The increased public awareness led, in part, to development of Public Law 94-142 (which includes a nondiscriminatory evaluation clause) and revised definitions of mental retardation. Specifically, the changes indicated a need for multiple assessment measures, including adaptive behavior testing, in determining the classification of mentally retarded students.

Recent definitions of mental retardation by the AAMD have specifically used the term adaptive behavior deficit to describe the criteria necessary for that classification (Grossman, 1977, 1983). Even so, the concept, if not the term adaptive behavior, has been considered in classification systems for a number of years. In fact, as early as 1941, Doll referred to "social incompetence" as one of the criteria necessary to identify an individual as retarded (in Reschly, 1984).

\section{PURPOSES OF ADAPTIVE BEHAVIOR ASSESSMENT}

Until recently, the vast majority of adaptive behavior measures were informal checklists or sequential lists of behavior that were inadequate for the assessment of such an important and conceptually difficult area (Taylor, 1982). In the past 10 years, however, more time, research, and effort have been expended in developing new adaptive behavior instruments. The more commonly used and widely accepted of these are norm-referenced tests. Norm-referenced tests are those that compare an individual's score to the performance of a standardization sample or normative group; a student's test performance is interpreted on the basis of its comparison to the performance of students on whom the test was normed.

Comparing a child's test performance to the norm is not necessarily the same as comparing the performance to "normal." Rather, the child's performance is being compared to the performance of individuals in the standardization sample. This sample might be a random group of nonhandicapped children across the United States for one test, and on another test it might be a trainable mentally retarded sample. The test's purpose partially determines the nature of the standardization sample. Clearly, the nature of the comparison group must be kept in mind when interpreting the results of adaptive behavior instruments.

In general, adaptive behavior instruments are used for two purposes. The first relates to identification of individuals who vary significantly from "normal" expectations in areas such as independent functioning and socialization. The applicable tests, sometimes referred to as descriptive (Cone \& Hawkins, 1977), are used to help make classification decisions as well as to identify overall problem areas and general strengths and weaknesses. Most descriptive adaptive behavior instruments are standardized on nonhandicapped individuals. 
Other adaptive behavior instruments yield much more specific information related to identification of educational/ instructional objectives. These types of tests, referred to by Cone and Hawkins as prescriptive, often are developed for and standardized on more severely handicapped children. Compared to descriptive tests, prescriptive tests typically include more specific and sequential items related to a fewer number of areas. Some instruments include both descriptive and prescriptive components.

Both types of instruments are important in the overall measurement of adaptive behavior, and individuals interested in assessing this area clearly should have a specific purpose in mind so that the appropriate type of instrument can be chosen. Unfortunately, many of the norm-referenced tests that are available have generally poor technical characteristics, including problems with reliability and validity. Reliability problems frequently surface as a function of the type of format used to gather the test information. Most adaptive behavior instruments rely on an interview with an informant (e.g., teacher, parent) to provide the test data. Often, the information obtained differs depending on the informant. For example, some evidence suggests that parents rate the same child higher than a teacher does (e.g., Mealor \& Richmond, 1980). Problems in validity occur for many reasons, not the least of which is the previously mentioned problem in defining and operationalizing the adaptive behavior construct.

\section{DESCRIPTION AND CRITIQUE OF ADAPTIVE BEHAVIOR INSTRUMENTS}

The instruments discussed here represent only a fraction of the many available adaptive behavior scales, but they are some of the newer, more frequently used scales, and they exemplify the various purposes of adaptive behavior assessments as well as the various formats of the tests themselves. Readers are encouraged to investigate the test manuals or relevant textbooks (e.g., Taylor, 1984) for more in-depth descriptions of these and other tests.

\section{Adaptive Behavior Inventory for Children (ABIC)}

The Adaptive Behavior Inventory for Children (Mercer, 1979) is a descriptive instrument that is a part of the battery of tests called the System Of Multicultural Pluralistic Assessment (SOMPA). One of the primary uses of the ABIC is to assist in the declassification of minority children labeled as mentally retarded. As such, it contains items that focus only on behaviors outside of the school environment. This test is the only adaptive behavior measure that has this feature. Information is obtained via a structured interview with a parent (usually the mother). The test is designed for use with 5- to 11-year-old children and yields standard scores $($ mean $=50$, standard deviation $=15)$.
Overall, the ABIC includes 242 items divided into six scales: family, community, peer relations, nonacademic school roles, earner/consumer, and self-maintenance. An example of a test item is: "Does (name of child) take telephone and other messages correctly and give them to the right person?"- to which the mother must respond "sometimes," "regularly," or "never." The test also includes a veracity scale used to determine whether the informant is artificially inflating the child's score.

Most of the research on the SOMPA centers on its use within the SOMPA model and its resulting effect on the declassification of minority special education students. For example, Reschly (1981) and Talley (1979) found that use of the ABIC greatly reduced the number of children in classes for mildly retarded students. Other research on the ABIC has focused on issues of validity-what type of skills the test is actually measuring. In general, there is a lack of supportive validity data to indicate what the test is measuring. Low correlations have been reported using achievement (Kazimour \& Reschly, 1981) and intelligence test results (e.g., Taylor, Ziegler, \& Partenio, 1985) as criterion measures.

\section{AAMD Adaptive Behavior Scale-School Edition (ABS-SE)}

The AAMD Adaptive Behavior Scale-School Edition (Lambert, Windmiller, Tharinger, \& Cole, 1981) is the most recent in a series of revisions of the original AAMD scale. The same general areas (called domains) and items have been included in the various revisions, but the standardization sample and the various scoring and interpretative procedures have been changed. For example, the ABS-SE was standardized on a school-based population (as opposed to an institutional group in the original test) and includes a scoring procedure that allows the separate comparison of an individual's performance to a regular education, an educable mentally retarded population, and a trainable mentally retarded population. The various purposes of the ABS-SE, as well as the procedural steps to follow, are shown in Figure 1.

This scale was designed for use with individuals age 3-16. The information about the child usually is provided on the test form by someone who is very familiar with his or her behavioral repertoire (e.g., teacher, parent). Part One of the instrument focuses on adaptive behavior such as self-help skills and personal independence; Part Two includes items related to maladaptive behavior.

Part One includes the following domains: Independent Functioning, Physical Development, Economic Activity, Language Development, Numbers and Time, Prevocational Activity, Self-Direction, Responsibility, and Socialization. In general, these domains contain items that require the informant to rate the child's behavior within a dependenceindependence continuum. For example, the item concerned 


\section{The ABS-SE Assessment Process}

Preparation: Gain experience with ABS-SE components. Training by someone familiar with the process is recommended."

Administration: Complete the Assessment Booklet by one of two methods:

First-person Assessment: A teacher or well-trained aide completes the scale by rating the student on each item.

Third-party Assessment: A teacher, psychologist, social worker or other trained person reads the scale items to someone unfamiliar with the scale, but who knows the student well, and records the reponses.

Scoring: Totals are obtained as each item, subdomain, and domain is completed in the Assessment Booklet.*

For Screening or Instruc-
tional Planning: The teacher
or aide completes the Instruc-
tional Planning Profile, converts
raw domain scores from the As-
sessment Booklet to percentile
scores, and plots a profile.
Results indicate whether
further evaluation is needed
and what areas of adaptive be-
havior require remediation.*

For Progress Evaluation: The teacher readministers the scale and compares results with those from a prior administration. Comparison indicates the extent of student progress and achievement of IEP goals and objectives. *

*For further information, refer to the Administration and Instructional Planning Manual. profile of factor scores. age group.** tional placement..*
For Diagnosis and Placement: The psychologist or other trained personnel completes the Diagnostic Profile using the Assessment Booklet to obtain scaled scores and a Comparison Score and plots a

Results indicate how a student's scores compare to scores of students in a similar

For IEP Evaluation: The teacher or psychologist readministers the scale and the IEP committee compares results with those from a prior administration. Comparison indicates the extent of student progress and appropriateness of educa-

**For further information, refer to the Diagnostic and Technical Manual.

Adapted from Adaptive Behavior Scale-School Edition by N. Lambert, M. Windmiller, D. Tharinger, \& M. Rosen (Washington, DC: American Association on Mental Deficiency, 1981). Used by permission.

\section{FIGURE 1}

\section{Purposes of and Procedural Steps in the ABS-SE}

with use of table utensils (in the Independent Functioning domain) requires that the child be rated on a 6-point scale from "feeds self with fingers or must be fed" (rating of 0 ) to "uses knife and fork correctly and neatly" (rating of 6).

The domains in Part Two are Aggressiveness, Antisocial vs. Social Behavior, Rebelliousness, Trustworthiness, Withdrawal vs. Involvement, Mannerisms, Appropriateness of Interpersonal Manners, Acceptability of Vocal Habits, Acceptability of Habits, Activity Level, Symptomatic Behavior, and Use of Medications. For each of these domains, several items are included in the form of behavioral descriptions (e.g., "uses threatening gestures"). The informant must indicate whether the examinee exhibits each behavior occasionally, frequently, or not at all.

Percentile ranks are available for all 23 domains. In addition, various domains have been collapsed into five factors:
Personal Self-Sufficiency, Community Self-Sufficiency, Personal-Social Responsibility, Social Adjustment, and Personal Adjustment. Scores from these five factors can be compared to the various groups (EMR, etc.) mentioned earlier.

The original ABS has been studied extensively. Most of this research has focused on technical characteristics of the instrument. Part Two has been scrutinized in particular. For example, the reliability has been questioned (Isett \& Spreat, 1979) and the norms criticized (McDevitt, McDevitt, \& Rosen, 1977). In addition, the fact that the severity of the maladaptive behavior is not taken into account can lead to misleading interpretations (Taylor, Warren, \& Slocumb, 1979). Little research data have been reported regarding the School Edition. The differences between the two tests would preclude any direct generalization of the previously noted research. Similarities of the tests, however, indicate that research is warranted with the School Edition.

\section{Balthazar Scales of Adaptive Behavior (BSAB)}

A good example of an instrument designed primarily for prescriptive reasons is the Balthazar Scales of Adaptive Behavior (Balthazar, 1976). Specifically, it was intended to be used for developing programs for severely and profoundly retarded individuals. Unlike most other scales, the BSAB uses a direct observational approach (rather than an interview) for obtaining the information.

The first section of the BSAB, the Scales of Functional Independence, measures the areas of eating, dressing, and toileting. The second section, the Scales of Social Adaptation, includes items measuring self-directed behavior, interpersonal behavior, verbal communication, play activities, and response to instructions. The items measuring these areas are broken down sequentially into much smaller steps than necessary for adaptive behavior instruments designed for more descriptive purposes. Thus, the items are frequently used as instructional objectives.

Research on the BSAB is generally lacking-probably because the normative use of the instrument is limited. Overall, the standardization sample was quite restricted, and the technical characteristics have not been explored adequately. In general, the test is used informally-almost as a checklist of possible instructional objectives.

\section{The Children's Adaptive Behavior Scale (CABS)}

The Children's Adaptive Behavior Scale (Richmond \& Kicklighter, 1980) is a unique instrument in that it tests the child directly rather than relying on interviews or observation. According to its authors, this feature was incorporated because of the lack of agreement when multiple raters (e.g., teachers and parents) provide information about a child's typical behavior. The scale was designed to be brief and to provide information that would be helpful to teachers in developing educational programs for 5- to 10 -year-old chil- 
dren. As such, the items are heavily weighted toward schooltype tasks.

Five separate areas or domains of adaptive behavior are measured: Language Development, Independent Functioning, Family Role Performance, Economic-Vocational Activity, and Socialization. A total score combines these five areas. Age-equivalents are available for each domain, as well as for the total score. Examples of the types of items include, "Name three kinds of animals that have four legs" (Language Development domain) and, "What would you do if you got lost in (nearest city or town)?" These items represent the more academic nature of the test as compared to other adaptive behavior instruments. They also indicate that it measures what the child would do as opposed to what he/she typically does.

Undoubtedly, the CABS will have a relatively high correlation with cognitive and academic measures. This is in contrast to the ABIC, for example, which has low correlations with those measures. The problems with the CABS relate to whether the items are different enough from other types of tests to warrant its addition in a psychometric battery and, if so, how the results can be used. Both of these issues relate to the validity of the test and would best be answered through empirical studies and continued use.

\section{Comprehensive Test of Adaptive Behavior (CTAB)}

The Comprehensive Test of Adaptive Behavior (Adams, 1984) uses a combination of direct observation and completion from memory to provide information. Unlike many other adaptive behavior tests, the CTAB has clearly identified criteria to indicate whether the subject has passed or

\section{8-(2) Stores refrigerated food}

Criterion: Student puts food needing refrigeration into airtight containers and places containers in refrigerator.

\section{8-(3) Prepares frozen juice}

Criterion: Student gets juice from freezer, opens can, and makes juice by mixing according to directions.

\section{8-(4) Freezes food}

Criterion: Student puts food needing freezing into the freezer.

\section{9-(1) Operates stove burners}

Criterion: Student turns appropriate burner on and adjusts to proper temperature. Student turns burner off when finished.

\section{9-(2) Makes instant hot beverages}

Criterion: Student makes instant hot beverages (for example, instant coffe or tea). failed an item. As a result, the information gathered is more reliable than that from many other scales. Figure 2 shows an example of the items and the criteria for passing.

There are over 500 items measuring the general areas of Self-Help Skills, House-Living Skills, Independent-Living Skills, Social Skills, Sensory and Motor Skills, and Language Concepts and Academic Skills. The CTAB was standardized using both retarded and nonretarded individuals so that various comparisons can be made.

Overall, the CTAB was appropriately named; it is a comprehensive instrument. As such, it has the possibility of being used for prescriptive purposes. Its use as a descriptive instrument is somewhat limited because of its length, however. For persons interested in a relatively fast, normative, descriptive instrument, the Normative Adaptive Behavior Checklist, designed to be used in conjunction with the CTAB, is available. This instrument contains 120 items that are answered in a yes-no format.

\section{The Scales of Independent Behavior (SIB)}

The Scales of Independent Behavior (Bruininks, Woodcock, Weatherman, \& Hill, 1984) is a relatively new instrument that can be used by itself or as a part of the WoodcockJohnson Psychoeducational Battery. By using the scales as a component of the total Battery, one can get a profile of an individual in the areas of cognitive ability, achievement, and interests, as well as adaptive behavior. The format of the SIB is highly structured (similar to the ABIC). The interviewer asks a series of questions such as, "Does eat with a fork by spearing the food when appropriate?" The respondent must indicate whether the individual can do

Test: After a meal and with containers available, say “ the (name of the food) away." , put

Test: Place student in front of freezer (frozen juice inside) with can opener and pitcher available. Say "___, there is some (name of the juice) in the freezer. Make some for me."

Test: Place student in front of a variety of food (some of which needs freezing, for example, ice cream). Say“ , put the food away."

Test: Place student in front of stove burners. Point to one of the burners and say “___ turn this burner on to ?___ (the temperature reading will vary depending on the stove available)."

Test: Place student in front of stove, pan (kettle), cup, and beverage (for example, instant coffee). Say “___, make a cup of (name of beverage)." 
that task without help or supervision "never or rarely," "about $1 / 4$ of the time," "about $3 / 4$ of the time," or "almost always."

Overall, the SIB is easy to administer and score, although the number of tables used to transform the raw score to derived scores is burdensome. It is designed for use with infants through adults and provides a variety of scores, including percentile ranks and standard scores.

The SIB can be given in its entirety or can be administered using one of several options. For example, a short form consisting of 32 items is available for screening purposes. The full battery contains 226 items broken down into the following areas: Gross Motor, Fine Motor, Social Interaction, Language Comprehension, Language Expression, Eating and Meal Preparation, Toileting, Dressing, Personal Self-Care, Domestic Skills, Time and Punctuality, Money and Value, Work Skills, and Home-Community Orientation. In addition, certain of the areas are combined to form Motor Skills, Social Interaction and Communication Skills, Personal Independence Skills, and Community Independence Skills clusters that can be administered and scored separately. A Problem Behavior Scale (see Figure 3) allows for the evaluation of maladaptive behavior.

In general, the SIB should be used for descriptive purposes, primarily for mildly/moderately retarded individuals. The items are neither sequential enough to use as a prescriptive instrument nor "low enough" to use with severely/profoundly retarded individuals. As with many new adaptive behavior tests, there is not enough research to date to explore its uses and limitations adequately.

\section{Vineland Adaptive Behavior Scales (VABS)}

Although the Vineland Adaptive Behavior Scales (Sparrow, Balla, \& Cicchetti, 1984) has a name similar to its predecessor, the Vineland Social Maturity Scale (VSMS), the two instruments are relatively dissimilar. Authors of the VABS acknowledge that Doll (author of the VSMS) made a significant contribution to the overall area of adaptive behavior, and they have used many of his concepts in designing the new instrument; however, Sparrow et al. have expanded this conception and developed a more comprehensive measure.

The test, designed for use with individuals aged 3 to 18 years, has three separate editions that can be used separately or in combination. The Interview Edition-Survey Form is most like the original Vineland and consists of 261 items. This edition provides an overall measure of adaptive behavior and can be helpful in identifying strengths and weaknesses. The Interview Edition-Expanded Form is composed of 541 items. This edition offers a prescriptive component to the VABS model because the items are more sequential and appropriate for use in developing Individual Education Plans. The Classroom Edition has a unique feature in that

\section{Problem Behavior: 8 Critical Areas Scored in 3 Valid and Useful Indexes}

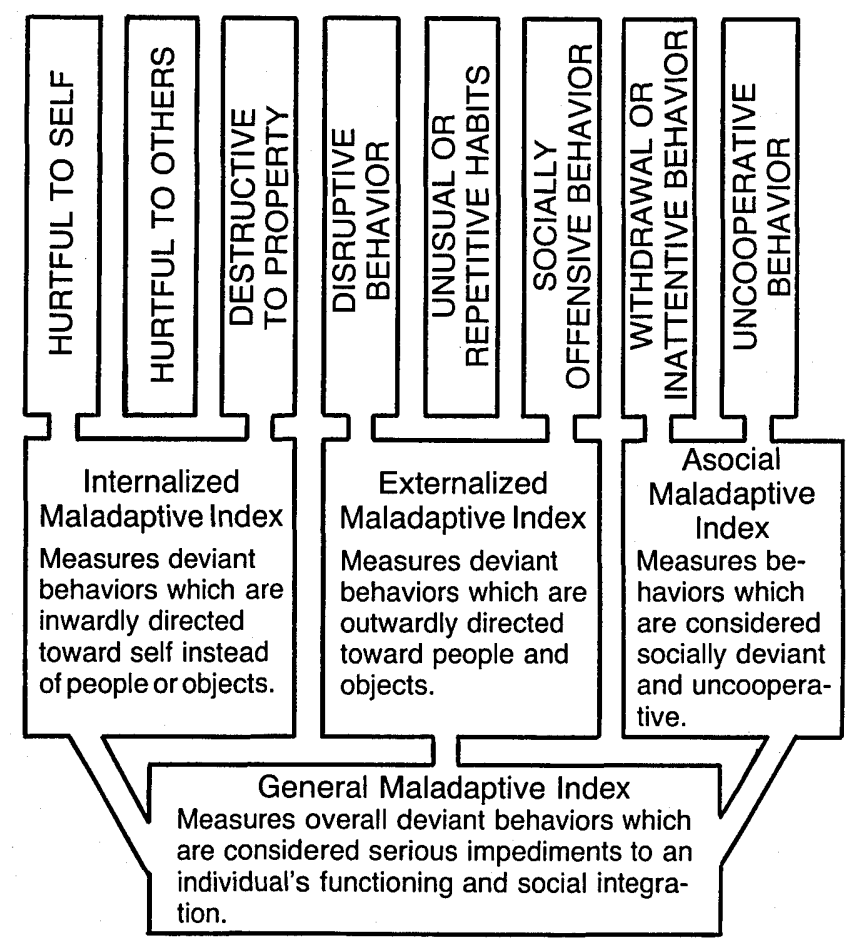

Adapted from R. Bruininks, R. Woodcock, R. Weatherman, \& B. Hill, The Scales of Independent Behavior (Allen, TX: DLM Teaching Resources, 1984). Used by permission.

\section{FIGURE 3}

\section{Problem Behavior Areas Measured by the SIB}

it allows the assessment of adaptive behavior within the classroom environment and is completed entirely by teachers. The two Interview Editions, in contrast, are administered in a semi-structured format with the parents or primary caregivers as the respondents. For example, the interviewer encourages the parent/caregiver to respond to the item, "Imitates sounds of adults immediately after hearing them." The interviewer then rates the response on a 0 (no, never) to a 2 (yes, usually) scale. A "don't know" (DK) and a "no opportunity" (NO) category are also available.

All three editions include five domains broken down into 12 subdomains. These are: Communication (Receptive, Expressive, Written), Daily Living Skills (Personal, Domestic, Community), Socialization (Interpersonal Relationships, Play and Leisure Time, Coping Skills), Motor Skills (Gross, Fine), and the Adaptive Behavior Composite (a combination of the other four domains). In addition, the two Interview Editions include an optional Maladaptive Behavior Domain focusing on undesirable behaviors that interfere with individual adaptive functioning.

A variety of scores are available from all three editions of the VABS: standard scores, national percentile ranks, 
stanines, and age equivalents. One particularly nice feature is a summary report for parents, presented in nontechnical language (see Figure 4).

Overall, the VABS promises to be one of the better adaptive behavior instruments published in the last 10 years. Its standardization was well-constructed, and its theoretical base is sound. The instrument can be used for both descriptive and prescriptive purposes so that an overall adaptive behavior package is available within the same instrument. In addition, a large portion of the standardization sample was also used in the norming of the Kaufman Assessment Battery for Children, so that measures of cognitive skills, achievement, and adaptative behavior are possible using the same comparison group.

\section{SUMMARY}

Most adaptive behavior instruments include items in the areas of independent functioning, self-help skills, sensory and motor skills, and social skills. Adaptative behavior scales are used for a variety of purposes. In some situations they are used to aid in decisions about special education classifications. In other situations they are used to help develop educational objectives for students receiving special education (particularly lower-ability students). Adaptive behavior instruments have been traditionally used with mentally retarded students or those suspected of being mentally retarded, although many have relevance for other types of students.

The measurement of adaptive behavior is age-related and, to a certain extent, culture-related. For example, adaptive behavior would be defined differently for a 3-7-year-old than for a 13-year-old child. Similarly, opportunities for exhibiting certain behaviors considered to be "adaptive" might be related to the geographic/socioeconomic/cultural background of an individual.

In general, the technical characteristics of many adaptive behavior instruments are limited. In particular, the reliability
The following chart describes more specific areas of adaptive behavior. Although scores such as percentile ranks and stanines are not given for these specific areas, the chart shows the areas in which your child's performance is above average, average, or below average when compared with the national norm group.

\begin{tabular}{|c|c|c|c|c|c|}
\hline & & & $\begin{array}{l}\text { Above } \\
\text { Average }\end{array}$ & Average & $\begin{array}{l}\text { Below } \\
\text { Average }\end{array}$ \\
\hline \multirow{3}{*}{ COMMUNICATION } & Receptive & What your child understands & & & \\
\hline & Expressive & What your child says & & & \\
\hline & Written & What your child reads and writes & & & \\
\hline \multirow{3}{*}{ DAILY LIVING SKILLS } & Personal & $\begin{array}{l}\text { How your child eats, dresses, and } \\
\text { practices personal hygiene }\end{array}$ & & & \\
\hline & Domestic & $\begin{array}{l}\text { What household tasks your child } \\
\text { performs }\end{array}$ & & & \\
\hline & Community & $\begin{array}{l}\text { How your child uses time, money, the } \\
\text { telephone, and job skills }\end{array}$ & & & \\
\hline \multirow{3}{*}{ SOCIALIZATION } & $\begin{array}{l}\text { Interpersonal } \\
\text { Relationships }\end{array}$ & How your child interacts with others & & & \\
\hline & $\begin{array}{l}\text { Play and Leisure } \\
\text { Time }\end{array}$ & $\begin{array}{l}\text { How your child plays and uses leisure } \\
\text { time }\end{array}$ & & & \\
\hline & Coping Skills & $\begin{array}{l}\text { How your child demonstrates respon- } \\
\text { sibility and sensitivity to others }\end{array}$ & & & \\
\hline \multirow[b]{2}{*}{ MOTOR SKILLS } & Gross & $\begin{array}{l}\text { How your child uses arms and legs for } \\
\text { movement and coordination }\end{array}$ & & & \\
\hline & Fine & $\begin{array}{l}\text { How your child uses hands and fingers } \\
\text { to manipulate objects }\end{array}$ & & & \\
\hline
\end{tabular}

General Summary

\section{Recommendations}

Adapted from Vineland Adaptive Behavior Scales by S. Sparrrow, D. Balla, \& D. Cicchetti. Reprinted by permission of American Guidance Service, Inc., Publishers' Bldg., Circle Pines, MN 55014. Copyrighted 1984. Rights reserved. 
of adaptive behavior instruments has been questioned, partially because of the interview format that is so commonly used. The validity of many of these tests has also been questioned, primarily because of the difficulty in defining the adaptive behavior construct.
These differing opinions regarding the definition and conceptualization of adaptive behavior have resulted in tests that are very different in terms of format as well as their purported use. Table 1 summarizes the instruments dicussed in this article.

TABLE 1

\section{SUMMARY PROFILE OF THE ADAPTIVE BEHAVIOR SCALES}

\section{Name}

Adaptive Behavior Inventory for
Children

AAMD Adaptive Behavior ScaleSchool Edition

Balthazar Scales of Adaptive

Behavior

Children's Adaptive Behavior

Scale

Comprehensive Test of Adaptive

Behavior

The Scales of Independent

Behavior

Vineland Adaptive Behavior

Scale, Birth -18 years

\section{REFERENCES}

Adams, G. (1984). The comprehensive test of adaptive behavior. Columbus, $\mathrm{OH}$ : Charles E. Merrill.

Balthazar, E. (1976). Balthazar scales of adaptive behavior. Palo Alto, CA: Consulting Psychologists Press.

Bruininks, R., Woodcock, R., Weatherman, R., \& Hill, B. (1984). The Scales of Independent Behavior. Allen, TX: DLM Teaching Resources.

Cone, J., \& Hawkins, R. (1977). Behavioral assessment: New directions in clinical psychology. New York: Brunner/Mazel.

Grossman, H. (1977). Manual on terminology and classification in mental retardation. Washington, DC: American Association on Mental Deficiency.

Grossman, H. (1983). Classification in mental retardation. Washington, DC: American Association on Mental Deficiency.

Guadalupe v. Tempe, Arizona School District, Civ. No. 71-435 (D. Ariz. 1972).

Isett, R., \& Spreat, S. (1979). Test-retest and interrater reliabilities of the AAMD Adaptive Behavior Scale. American Journal of Mental Deficiency, 84, 93-95.

Kazimour, K., \& Reschly, D. (1981). Investigation of the norms and concurrent validity for the Adaptive Behavior Inventory for Children. American Journal of Mental Deficiency, 85, 512520 .

Lambert, N., Windmiller, M., Tharinger, D., \& Cole, L. (1981). $A A M D$ adaptive behavior scale-school edition. Washington, DC: American Association on Mental Deficiency.

McDevitt, S., McDevitt, S., \& Rosen, M. (1977). Adaptive behavior scale-Part two: A cautionary note and suggestions for revisions. American Journal of Mental Deficiency, 82, 210-212.

Mealor, D., \& Richmond, B. (1980). Adaptive behavior: Teachers and parents disagree. Exceptional Children, 46, 386-389.

Mercer, J. (1973). Labeling the mentally retarded: Clinical and
Type

Source of Information

Descriptive

Structured Interview

Descriptive/Prescriptive

Semi-structured interview

Prescriptive

Observation

Descriptive

Descriptive-Prescriptive

(with NABC)

Descriptive

Direct administration to child

Completion from memory; observation

Structured interview

Semi-structured interview

social system perspectives on mental retardation. Berkeley, CA: University of California Press.

Mercer, J. (1979). System of multicultural pluralistic assessment. New York: Psychological Corp.

Reschly, D. (1981). Evaluation of the effects of SOMPA measures on classification of students as mildly mentally retarded. American Journal of Mental Deficiency, '86, 16-20.

Reschly, D. (1984). Best practices in conceptualizing adaptive behavior. In J. Grimes \& A. Thomas (Eds.), Best practices in school psychology. Stratford, CT: NASP Publications.

Richmond, B., \& Kicklighter, R. (1980). The children's adaptive behavior scale. Atlanta: Humanics, Ltd.

Sparrow, S., Balla, D., \& Cicchetti, D. (1984). Vineland Adaptive Behavior Scales. Circle Pines, MN: American Guidance Service.

Talley, R. (1979). Evaluating the effects of implementing the System of Multicultural Pluralistic Assessment: A qualitative perspective. School Psychology Digest, 8, 71-78.

Taylor, R. (1982). Assessment. In L. Sternberg \& G. Adams (Eds.), Educating severely and profoundly handicapped students. Rockville, MD: Aspen Systems.

Taylor, R. (1984). Assessment of exceptional students: Educational and psychological procedures. Englewood Cliffs, NJ: PrenticeHall.

Taylor, R., Warren, S., \& Slocumb, P. (1979). Categorizing behavior in terms of severity: Considerations for part two of the Adaptive Behavior Scale. American Journal of Mental Deficiency, 83, 411-414.

Taylor, R., Ziegler, E., \& Partenio, I. (in press). An empirica investigation of the Adaptive Behavior Inventory for Children. Psychological Reports.

Witt, J., \& Martens, B. (1984). Adaptive behavior: Tests and assessment issues. School Psychology Review, 13, 478-484.

The author extends a special thanks to Yvette Taylor for her careful technical editing of this manuscript. 